\title{
Hemangioma capilar periocular en 2018: curso natural, indicaciones para el tratamiento y opciones terapéuticas
}

\section{Periocular capillary hemangioma in 2018: natural course, indications for treatment and therapeutic options}

\author{
Carlos Fleitman-Broder* \\ Centro Médico ABC, Ciudad de México, México
}

\begin{abstract}
Resumen
Los hemangiomas capilares son el tumor de párpado y órbita más común en la infancia. La mayoría de estos tumores no requieren tratamiento, gracias a que involucionan espontáneamente sin dejar secuelas permanentes; sin embargo, en muchos casos, el tratamiento es necesario, por ejemplo, en el caso de lesiones ambliogénicas o que pongan en riesgo la salud ocular. El tratamiento de este tipo de lesiones ha evolucionado a lo largo de los años, desde la escisión quirúrgica, pasando por el láser y los tratamientos con esteroides, todos ellos con diferentes grados de éxito y efectos secundarios. En los últimos años, gracias a un hallazgo casual de Leaute-Labreze, et al., el tratamiento de estas lesiones ha sufrido una revolución. Ahora, en el arsenal terapéutico para este tipo de lesiones contamos con fármacos betabloqueadores, que han demostrado ser altamente efectivos y con un rango de seguridad mucho mayor que los tratamientos previamente utilizados. Hoy en día existen también reportes de casos que demuestran éxito en el tratamiento de estas lesiones con aplicación tópica de esta familia de medicamentos, lo que disminuye los efectos secundarios. Al contar con modalidades terapéuticas más seguras y eficaces, es posible tratar hemangiomas que en el pasado solamente se hubieran observado, así, se disminuyen las secuelas cosméticas y el estigma social que estos niños viven al tener una lesión de esta naturaleza en el área facial.
\end{abstract}

Palabras clave: (Tratamiento de) Neoplasias palpebrales. (Tratamiento de) Hemangioma capilar. (Tratamiento de) Neoplasias orbitales. (Uso terapéutico) Propanolol. (Tratamiento de) Neoplasias faciales.

\begin{abstract}
Capillary hemangiomas are one of the most common benign orbital and eyelid childhood tumors. Many of these tumors do not require treatment because they regress spontaneously without leaving sequelae; however, treatment is necessary in many cases, such as amblyogenic or eye-threatening lesions. Treatment of this kind of lesions has evolved from surgical excision to laser and steroids, all of them successful in different ways and with a range of side effects. In the last few years treatment has been revolutionized due to a casual finding made by Leaute-Labreze et al. Nowadays we use beta-blockers that have proved to be highly effective and with a much safer profile than previously used drugs. Several case reports have shown success by treating these lesions with topical beta-blockers. With this safer and more effective treatment, we can currently
\end{abstract}

\section{Correspondencia:}

${ }^{*}$ Carlos Fleitman-Broder

Av. Vasco de Quiroga,

4001, Torre A, $5 .^{\circ}$ piso, Cons. 2

Col. Santa Fe, Del. Cuajimalpa

Fecha de recepción: 26-09-2018

Fecha de aceptación: 26-02-2019

Ciudad de México, México

E-mail: cfleitman@gmail.com

DOI: 10.24875/RMO.M19000069

0187-4519/○ 2019 Sociedad Mexicana de Oftalmología. Publicado por Permanyer México. Este es un artículo Open Access bajo la licencia CC BY-NC-ND (http://creativecommons.org/licenses/by-nc-nd/4.0/).

Disponible en internet: 01-05-2019 Rev Mex Oftalmol. 2019;93(3):142-148 www.rmo.com.mx 
treat this lesions that in the past were just kept under observation, diminishing the cosmetic sequelae and the social stigma of patients having such lesions in their facial area.

Key words: Eyelid neoplasms (therapy). Hemangioma, capillary (therapy). Orbital neoplasms (therapy). Propranolol (therapeutic use). Facial neoplasms (therapy).

\section{Introducción}

Los hemangiomas capilares son el tumor de párpado y órbita más común en los infantes. Se componen de capilares anormales con proliferación de células endoteliales ${ }^{1-4}$.

La presentación más común del hemangioma es la de una tumoración de color rojo y aspecto multilobulado (Fig. 1). Si este es superficial o de color azulado (Fig. 2), si es subdérmico, o si es una masa en la zona anterior de la órbita, generalmente aumenta de tamaño durante los primeros años de vida, para después involucionar espontáneamente. La mayoría de los hemangiomas no plantean ninguna amenaza al desarrollo visual, pero si son lesiones grandes pueden causar ambliopía debido a ptosis mecánica, astigmatismo, proptosis grave con queratopatía por exposición y/o dañar el nervio óptico, dependiendo de la localización del tumor. El desarrollo visual comienza muy temprano en la infancia, y la privación del estímulo visual durante el primer año de vida ha demostrado ser perjudicial para el desarrollo visual. Los estudios han demostrado que la obstrucción total del eje visual por un periodo de 1 semana entre el nacimiento y la edad de 1 año puede dar lugar a ambliopía; por lo tanto, la identificación y el tratamiento apropiado y oportuno de este tipo de lesiones es de suma importancia ${ }^{1-3,5,6}$.

El aspecto histopatológico de este hamartoma vascular varía con su fase clínica. En la fase proliferativa temprana, el tumor consiste sobre todo en células endoteliales, y los espacios vasculares son raros, así mismo, contiene numerosos mastocitos. En los tumores más maduros se observan espacios vasculares más grandes, con menor número de células endoteliales (Fig. 3). El tumor no se encapsula y, generalmente, no tiende a infiltrar las estructuras circundantes. En la fase involutiva hay a menudo deposición del tejido fibroso y adiposo alrededor y dentro de la lesión ${ }^{3}$.

\section{Patogénesis}

Los hemangiomas son los tumores más comunes de la infancia, con una prevalencia entre infantes del 5 al $12 \%$ y un predominio en el sexo femenino de 3 a 1. La

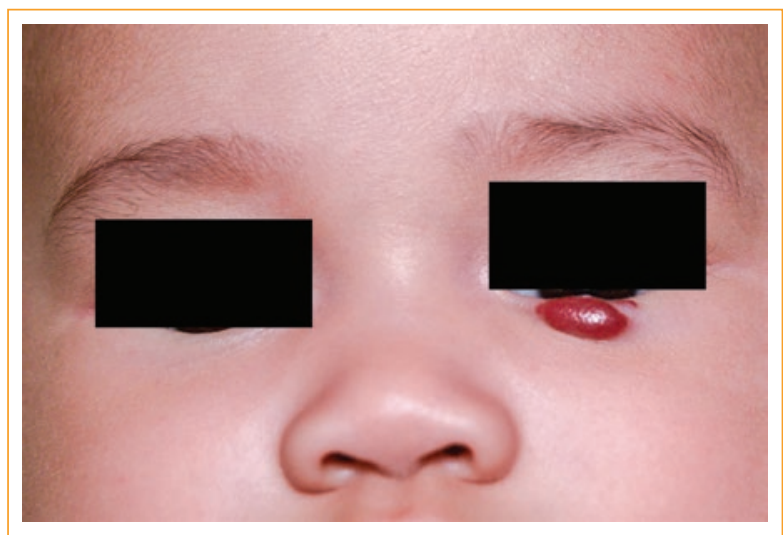

Figura 1. Hemangioma capilar superficial, párpado inferior izquierdo.

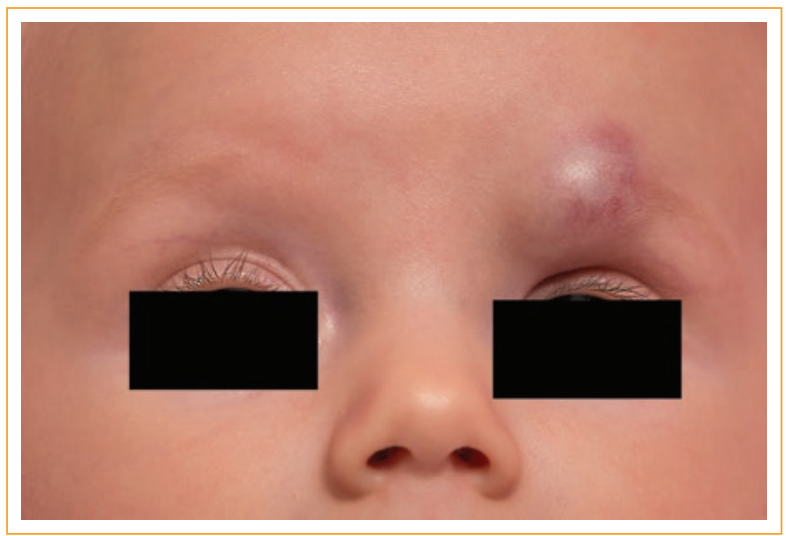

Figura 2. Hemangioma capilar profundo de la ceja izquierda.

prevalencia de los hemangiomas periorbitales y del párpado es del 1 hasta el 3\%. No se ha encontrado ningún patrón de herencia familiar ${ }^{1-3}$.

No existe una clasificación de los hemangiomas; sin embargo, muchos autores los clasifican basándose en el grado de profundidad. Los hemangiomas superficiales o capilares, designados históricamente «hemangiomas en fresa", se desarrollan a partir de la dermis papilar, no como los hemangiomas profundos o cavernosos, situados dentro de la dermis reticular y el tejido subcutáneo ${ }^{5}$. 


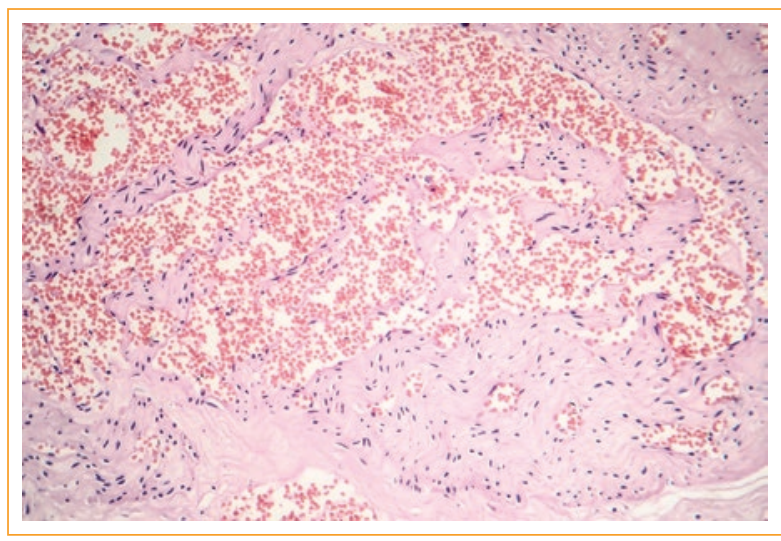

Figura 3. Corte histopatológico de un hemangioma capilar con proliferacion endotelial y múltiples espacios vasculares $(\mathrm{HE}, 40 \mathrm{x})$.

Las complicaciones sistémicas asociadas a los hemangiomas capilares incluyen el síndrome de PHACE (malformaciones cerebrales de la fosa Posterior, Hemangiomas, anomalías Arteriales, Coartación de la aorta y defectos cardíacos, y anormalidades oculares [Eye abnormalities]), así como la afectación visceral por estas lesiones ${ }^{1-3,5}$.

La patogénesis exacta de los hemangiomas no está clara, pero los estudios sugieren que la angiogénesis y la vasculogénesis desempeñan un papel importante. Se especula que existe un defecto del desarrollo durante las semanas 8 a 10 de gestación que provoca la formación de vasos anormales por parte de un precursor endotelial, lo que contribuye al proceso de angiogénesis patológica ${ }^{3}$.

Durante la fase proliferativa posnatal, este tumor expresa numerosos marcadores celulares de angiogénesis (factor de crecimiento endotelial vascular [VEGF], factor de crecimiento de fibroblastos básico [BFGF], colagenasa de tipo IV). Así mismo, se ha encontrado una falta de expresión de sustancias inhibidoras de la angiogénesis. La maduración vascular incompleta observada en infantes prematuros también ha sido asociada con una mayor incidencia de hemangiomas capilares ${ }^{5}$.

Una de las teorías más aceptadas sobre el origen de los hemangiomas capilares especula que estos se originan de tejido angioblástico placentario, fenotípicamente comparten algunas características microvasculares con el tejido placentario. North, et al. identificaron marcadores inmunohistoquímicos comunes en el tejido placentario y muestras histopatológicas de hemangiomas capilares, incluyendo transportador de glucosa 1 (GLUT1), receptor de Fc de inmunoglobulina tipo II (FcgRII), merosina y antígeno Lewis $Y^{5,7}$. Barnes, et al. evidenciaron la similitud en los transcriptomas de los hemangiomas y los del tejido placentario, con lo que se reforzó, desde un punto de vista genético, la teoría de origen placentario del hemangioma capilar ${ }^{5,8}$.

Uno de los factores de riesgo para desarrollar hemangiomas capilares es la toma de muestras de vellosidades coriónicas que podría potencialmente sembrar tejido placentario metastásico en áreas remotas, induciendo así la formación de este tipo de tumores ${ }^{5}$.

\section{Características clínicas y diagnóstico}

Aproximadamente un tercio de los hemangiomas capilares está presente al nacimiento, y todos habrán aparecido a la edad de 6 meses. La aparición del tumor suele ser precedida por un eritema cutáneo leve ${ }^{3}$.

El crecimiento rápido dura aproximadamente de 3 a 6 meses, es seguido por un período de estabilización y después, generalmente, de regresión. A los 7 a 9 años, el 76 a $90 \%$ de hemangiomas han involucionado sin ningún tratamiento. Sin embargo, en el 40 a 50\% de los casos, perduran secuelas de la lesión, como vasos telangectásicos, tejido fibroso y graso residual y/o cicatrización anormal1-3.

Los hemangiomas capilares se sitúan lo más comúnmente en el párpado superior o la órbita superior. Su aspecto varía según la profundidad de la lesión; las lesiones cutáneas superficiales tienen un aspecto lobulado de color rojo, han sido descritos comúnmente como «hemangiomas en fresa», y pueden crecer y tomar una coloración azulada durante el llanto. Los hemangiomas subcutáneos se presentan como una masa suave de color azulado, y los orbitales se presentan con signos y síntomas correspondientes al de una masa a nivel orbital (proptosis, neuropatía óptica compresiva, etc. $)^{1-3,5}$.

El desarrollo de ambliopía secundaria a oclusión del eje visual, astigmatismo, anisometropía, estrabismo por compresión del globo ocular o extensión del tumor al espacio retrobulbar es la complicación más común, con una incidencia que va del 43 al $60 \%$.

El diagnóstico puede ser establecido clínicamente en la mayoría de los casos, sin embargo, en algunas ocasiones pueden ser necesarios estudios de imagen para descartar afectación visceral o neoplasias agresivas.

El diagnóstico diferencial incluye angioblastoma, neuroblastoma, rabdomiosarcoma, fibrosarcoma, hemangiopericitoma y miofibrosis infantil ${ }^{5}$.

El ultrasonido Doppler puede ser de utilidad en los casos en los que existe una duda diagnóstica, al demostrar 
un patrón de alto flujo, asimetría e irregularidad acústica, a diferencia de los tumores sólidos y malformaciones vasculares que exhiben un patrón de bajo flujo.

En la tomografía axial computariza, el hemangioma capilar muestra una masa homogénea que se refuerza con la inyección de medio de contraste, en algunas ocasiones es posible demostrar la presencia de los vasos sanguíneos que nutren al tumor. La resonancia magnética muestra una lesión lobulada con una intensidad de señal intermedia, la cual es isointensa con los músculos extraoculares en T1 e hiperintensa en $\mathrm{T} 2^{3,5}$.

En raras ocasiones es necesario realizar una biopsia para obtener una confirmación histopatológica de la lesión.

\section{Tratamiento, ¿cuándo y cómo tratar?}

Los hemangiomas periorbitales y palpebrales están asociados a complicaciones que pueden tener un gran efecto en la calidad de la visión si no se tratan a tiempo. En muchos casos, la observación y manejo expectante son suficientes, ya que estas lesiones involucionan espontáneamente.

La presencia de una lesión ambliogénica es la indicación más común de tratamiento, otras indicaciones menos comunes son crecimiento rápido del tumor, neuropatía óptica compresiva, y proptosis con queratopatía por exposición ${ }^{1-6,9}$.

La ansiedad de los padres por la presencia del defecto cosmético que estas lesiones pueden llegar a provocar puede presentar un reto terapéutico importante que no debe dejarse a un lado. Otras de las indicaciones relativas para el tratamiento son ulceración, sangrado e infección.

Existen muchas estrategias diferentes para el tratamiento de los hemangiomas capilares. Sin embargo, debido a que estas lesiones sufren una regresión espontánea y los tratamientos existentes no están exentos de efectos secundarios, el tratamiento suele reservarse para las lesiones con un potencial de complicaciones.

Los tratamientos más comúnmente utilizados hasta hace pocos años incluyen corticoesteroides, láser y resección quirúrgica. Algunos tratamientos, como crioterapia, radiación y electrolisis, hoy en día son prácticamente obsoletos. El uso de inmunomoduladores como ciclofosfamida e interferón alfa se reserva solamente como tratamiento de segunda línea en lesiones que ponen en riesgo la vida o la visión. Finalmente, en los últimos años, el tratamiento con betabloqueadores ha tenido cada vez más aceptación y excelentes resultados, después del descubrimiento incidental de
Leaute-Labreze, et al. de la regresión de los hemangiomas capilares en pacientes que recibieron propanolol por indicación cardiovascular ${ }^{10}$.

\section{Terapia con corticoesteroides}

Los corticoesteroides se han utilizado en el tratamiento de hemangiomas capilares durante más de cuatro décadas. Su mecanismo de acción para lograr involución de la lesión aún sigue sin entenderse. El tratamiento puede administrarse por vía oral, intralesional y/o tópica ${ }^{5,11}$.

Hasta hace poco tiempo, la inyección intralesional de corticoesteroides era considerado el tratamiento de primera línea para lesiones pequeñas de bajo riesgo. El tratamiento se basa en 1-2 ml intralesionales de una mezcla de triamcinolona $(40 \mathrm{mg} / \mathrm{ml})$ más betametasona $(6 \mathrm{mg} / \mathrm{ml})$. Una reducción del astigmatismo de hasta 1.4 D, o más, se ha reportado en el 53.8 a $66 \%$ de los pacientes; sin embargo, efectos secundarios cutáneos, como hipopigmentación y atrofia grasa se ha reportado hasta en un $70 \%$ de los pacientes, así mismo, también se han reportado casos aislados de necrosis palpebral, obstrucción de la arteria central de la retina e hipertensión ocular ${ }^{5,11}$.

Para el tratamiento de hemangiomas capilares de gran tamaño se puede considerar el tratamiento con corticoesteroides sistémicos. La regresión de la lesión se observa en 2 semanas en el $30 \%$ de los casos; sin embargo, en muchas ocasiones es necesario prolongar el tratamiento más allá de 8012 semanas. Boon, et al. reportaron una serie de 62 pacientes que recibieron dosis diarias menores a $3 \mathrm{mg} / \mathrm{kg} / \mathrm{día}$ durante 1 mes con reducción progresiva de la dosis a lo largo de 2 a 3 meses. La mayoría de los efectos secundarios fueron temporales, mientras el paciente se encontraba bajo tratamiento, un tercio de los pacientes sufrieron retraso en el crecimiento; sin embargo, el $91 \%$ lo recuperaron en los 2 años posteriores a la suspensión de los esteroides. Se reportaron cambios en la personalidad durante el tratamiento en el $29 \%$ de los pacientes ${ }^{12}$.

El problema más importante con el tratamiento de corticoesteroides es la mala respuesta al tratamiento. En la literatura se reporta hasta un 11 a $30 \%$ de casos que no responden, así mismo, se han reportado casos en los que la lesión recurre una vez suspendido el tratamiento $0^{5,6,11,13}$.

\section{Tratamiento con láser}

Aunque los láser de tipo $\mathrm{Nd}$ : YAG y de $\mathrm{CO}_{2}$ han sido utilizados como tratamiento para el hemangioma 
capilar con resultados relativamente aceptables, hoy en día el láser pulsado es el láser de elección para este tipo de tratamientos $\mathrm{s}^{1,2,3,5}$.

La longitud de onda ideal para el tratamiento de hemangiomas capilares es de 585 a 600 nanometros ${ }^{5}$.

La mayoría de los expertos recomiendan el uso de tratamiento láser solamente en las etapas de proliferación temprana o de regresión tardía debido a la poca penetración de estos. Cuando la lesión es plana en su estadio temprano, el láser pulsado se puede utilizar a intervalos de 4 a 6 semanas. Así mismo, el tratamiento con láser puede utilizarse para las telangiectasias residuales secundarias a un hemangioma que involuciono ${ }^{5}$.

\section{Tratamiento quirúrgico}

El tratamiento quirúrgico puede ser una buena opción en pacientes con una lesión subcutánea con bordes bien definidos, o cuando los demás tratamientos han fallado $0^{3,13}$.

Las indicaciones para el tratamiento quirúrgico son falta de respuesta al tratamiento médico, crecimiento rápido de la lesión y deformidad orbital y/o palpebral graves. Muchos autores han reportado buenos resultados cosméticos y/o funcionales con el tratamiento quirúrgico ${ }^{3,13}$.

Los principales efectos adversos de la excisión quirúrgica son: cicatrización, hemorragia y complicaciones relacionadas con la anestesia general. Existen reportes aislados de lesiones que requirieron transfusión sanguínea intraoperatoria ${ }^{13}$.

El principal objetivo de la excisión quirúrgica temprana es la prevención de la ambliopía causada por el astigmatismo o depravación visual. Mientras que el tratamiento quirúrgico tardío tiene un objetivo cosmético.

\section{Inmunoterapia y quimioterapia}

Los inmunomoduladores son agentes terapéuticos potentes con efectos secundarios graves, por lo tanto, se reservan para lesiones que ponen en peligro el ojo o la vida.

La ciclofosfamida con o sin esteroides se ha utilizado con buenos resultados ${ }^{4}$. Estudios recientes también han demostrado la efectividad del interferón alfa 2 (IFN- $\alpha 2)$ para el tratamiento del hemangioma capilar ${ }^{3}$. Los efectos secundarios de estos medicamentos incluyen mielosupresión, hepatotoxicidad y neurotoxicidad. Los efectos a largo plazo del IFN- $\alpha 2$ en el cerebro en desarrollo son desconocidos.

\section{Antagonistas betaadrenérgicos}

Leaute-Labreze, et al. notaron que la administración de propanolol sistémico en un niño con cardiomiopatía hipertrófica provocó la regresión de un hemangioma capilar nasal. Estos mismos autores demostraron un total de 11 casos de hemangiomas infantiles tratados exitosamente con $2 \mathrm{mg} / \mathrm{kg} / \mathrm{día}$ de propanolol sistémico por un periodo de hasta 9 meses $^{10}$.

Posterior a este hallazgo, se han publicado un gran número de reportes que han demostrado regresiones importantes de hemangiomas en diferentes partes del cuerpo ${ }^{4,9,14}$.

El mecanismo de acción del propanolol en la reducción de los hemangiomas no se ha dilucidado, pero las posibles explicaciones incluyen vasoconstricción, que es visible inmediatamente como un cambio de coloración y mayor suavidad a la palpación. Otros factores que se han propuesto como mecanismo de acción es la disminución de la expresión de diferentes factores angiogénicos, como VEGF y BFGF, así como un aumento en la apoptosis de células endoteliales capilares ${ }^{4,5,10}$. El propanolol tiene un perfil de seguridad y efectos secundarios bien establecidos. Existe una gran experiencia de uso en pacientes pediátricos con enfermedad cardiovascular e hipertensión arterial. A pesar de que se han demostrado efectos secundarios graves en infantes que fueron expuestos a betabloqueadores in utero, la exposición posnatal parece ser segura y libre de efectos secundarios graves.

Las reacciones secundarias adversas de los betabloqueadores incluyen bradicardia, hipotensión, hipoglucemia, reflujo gastroesofágico, fatiga y broncoespasmo $0^{5,15}$.

Una historia clínica y exploración física enfocados en enfermedades cardiovasculares y respiratorias son necesarias antes de la administración del tratamiento.

El protocolo más ampliamente utilizado para este tratamiento es ${ }^{16}$ :

- Ecocardiografía de base y una hospitalización de $48 \mathrm{~h}$ o visitas para control de signos vitales pretratamiento.

- Administración de propanolol $0.16 \mathrm{mg} / \mathrm{kg}$ cada $8 \mathrm{~h}$ como dosis de inicio.

- Aumento de la dosis hasta un máximo de $0.67 \mathrm{mg} / \mathrm{kg}$ cada $8 \mathrm{~h}(2 \mathrm{mg} / \mathrm{kg} / \mathrm{día})$ si los signos vitales y la glucemia lo permiten.

- El tratamiento debe mantenerse durante la fase proliferativa del hemangioma o hasta que no exista mejoría visible.

- Disminución gradual de la dosis por un periodo de 2 semanas.

Haider, et al. publicaron en 2010 un protocolo para el tratamiento con propanolol oral sin la necesidad de 


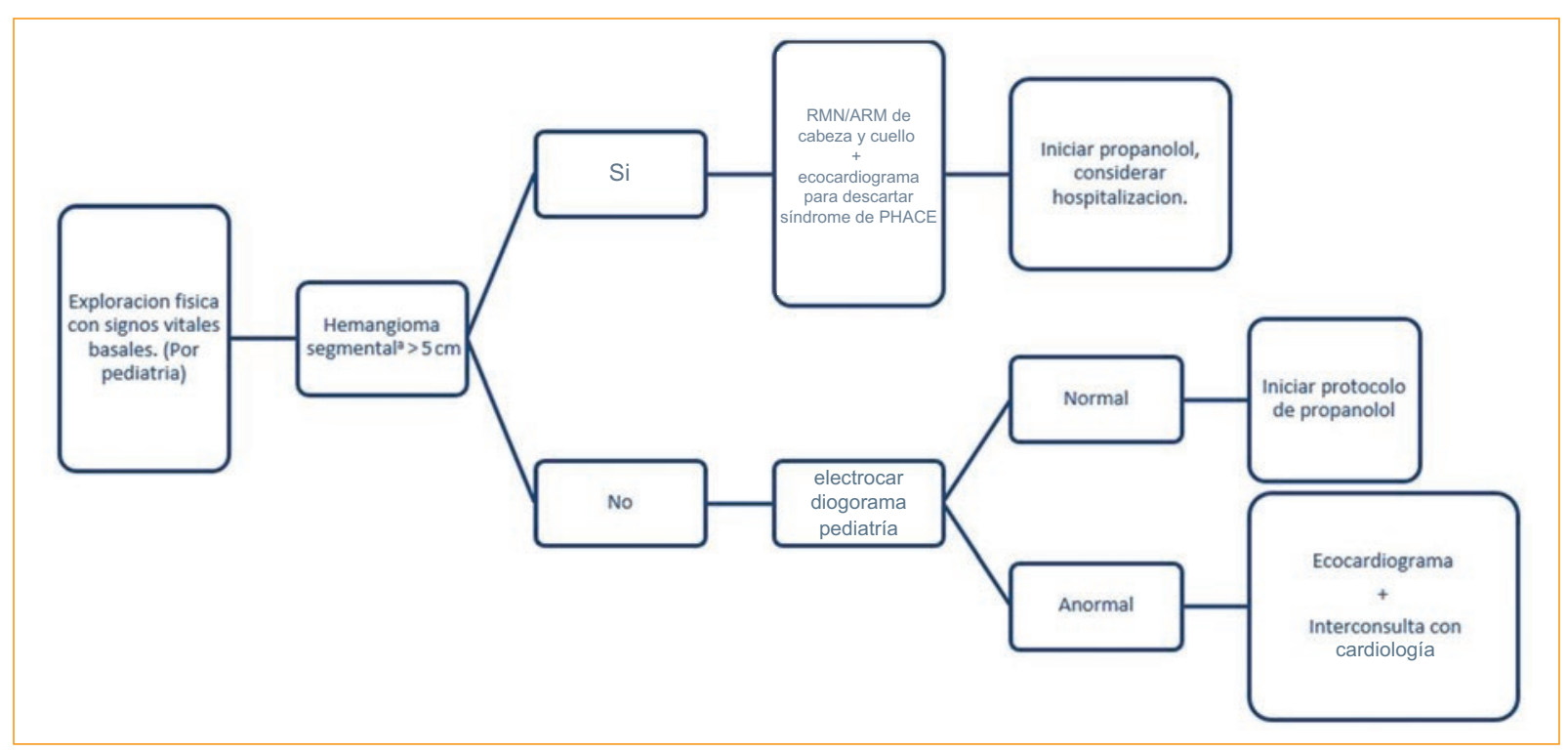

Figura 4. Protocolo para la evaluación de pacientes previos al tratamiento con propanolol (modificado de Haider, et al. $\left.{ }^{16}\right)$.

aLesión lobulada con configuración geográfica.

PHACE: malformaciones cerebrales de la fosa Posterior, Hemangiomas, anomalías Arteriales, Coartación de la aorta y defectos cardíacos, y anormalidades oculares (Eye abnormalities); RMN: resonancia magnética nuclear.

hospitalización (Fig. 4) en 17 pacientes, y lograron detener el crecimiento o disminuir el tamaño de todas las lesiones tratadas. Solamente se presentaron efectos secundarios, que fueron leves, en 6 de estos pacientes, y no hubo necesidad de suspender el tratamiento en ningún paciente ${ }^{17}$ (Tabla 1).

Guo, el al. reportaron por primera vez la efectividad del uso de solución de timolol $0.5 \%$ tópico aplicado directamente sobre la superficie de la lesión ${ }^{18}$. Desde entonces varios estudios han demostrado la efectividad del tratamiento tópico con solución de timolol $0.5 \%$ o gel al $0.1 \% 3$ o 4 veces al día, con un excelente perfil de seguridad. Así mismo, se ha demostrado que los hemangiomas capilares que mejor responden a este tratamiento son los superficiales y aquellos que se encuentran en fase proliferativa ${ }^{16,18-24}$.

En la mayoría de los centros de referencia, los betabloqueadores tópicos o sistémicos, dependiendo de las características de la lesión, son hoy en día el tratamiento de primera línea para este tipo de lesiones.

\section{Conclusiones}

Los hemangiomas capilares son los tumores perioculares más comunes en la infancia y representan un factor de riesgo importante para la pérdida de visión y desarrollo de ambliopía a pesar de que la historia
Tabla 1. Protocolo para la administración de propanolol (modificado de Haider, et al. ${ }^{16}$ )

\begin{tabular}{|c|c|}
\hline Suspensión & $20 \mathrm{mg} / 5 \mathrm{ml}$ o $40 \mathrm{mg} / 5 \mathrm{ml}$ \\
\hline Dosis & $\begin{array}{l}0.5 \mathrm{mg} / \mathrm{kg} / \text { día dividido en } 3 \text { dosis durante } \\
2-3 \mathrm{días} \\
1.0 \mathrm{mg} / \mathrm{kg} / \text { día dividido en } 3 \text { dosis durante } \\
2-3 \mathrm{días} \\
1.5 \mathrm{mg} / \mathrm{kg} / \text { día dividido en } 3 \text { dosis durante } \\
2-3 \mathrm{días} \\
2.0 \mathrm{mg} / \mathrm{kg} / \text { día dividido en } 3 \text { dosis (final) }\end{array}$ \\
\hline Monitorización & $\begin{array}{l}\text { Medir presión arterial + ritmo cardíaco } 24 \text { h } \\
\text { después de cada cambio de dosis }\end{array}$ \\
\hline Cesamiento & $\begin{array}{l}\text { Disminuir la dosis gradualmente durante } \\
2 \text { semanas }\end{array}$ \\
\hline
\end{tabular}

natural de la enfermedad es la de tener una regresión espontánea.

La identificación y tratamiento de las lesiones ambliogénicas es de extrema importancia, así como el seguimiento de cerca de los casos en los que el riesgo de evolucionar hacia una lesión ambliogénica sea alto.

Actualmente, los antagonistas betaadrenérgicos han revolucionado el tratamiento de estas lesiones, dando una invaluable opción, con excelentes resultados y perfil de seguridad al arsenal terapéutico con el que contamos para este tipo de lesiones. 
Hoy en día, el estándar de oro para el tratamiento de los hemangiomas capilares es, sin duda, el tratamiento con betabloqueadores, ya sea en su modalidad sistémica o tópica, dependiendo del tipo de lesión. Al contar con modalidades terapéuticas más seguras y eficaces, es posible tratar lesiones que en el pasado solamente se hubieran observado, así se disminuyen las secuelas cosméticas y el estigma social que estos niños viven al tener una lesión de esta naturaleza en el área facial.

\section{Conflicto de intereses}

El autor declara no tener conflicto de intereres con ninguno de los contenidos o medicamentos mencionados en este artículo.

\section{Responsabilidades éticas}

Protección de personas y animales. Los autores declaran que para esta investigación no se han realizado experimentos en seres humanos ni en animales.

Confidencialidad de los datos. Los autores declaran que han seguido los protocolos de su centro de trabajo sobre la publicación de datos de pacientes.

Derecho a la privacidad y consentimiento informado. Los autores han obtenido el consentimiento informado de los pacientes y/o sujetos referidos en el artículo. Este documento obra en poder del autor de correspondencia.

\section{Bibliografía}

1. Hoyt C, Taylor D. Pediatric Ophthalmology and Strabismus. $3^{\text {rd }}$ ed. Philadelphia: Elsevier Saunders; 2005

2. Nelson LB, Olitsky SE. Harley's Pediatric Ophthalmology. $5^{\text {th }}$ ed. Philadelphia: Lippincott Williams \& Wilkins; 2005.

3. Shields JA, Shields CL. Eyelid, Conjunctival, and Orbital Tumors. $2^{\text {nd }}$ ed. Philadelphia: Lippincot Williams \& Wilkins; 2008.
4. Fabian ID, Ben-Zion I, Samuel C, Spierer A. Reduction in astigmatism using propranolol as first-line therapy for periocular capillary hemangioma. Am J Ophthalmol. 2011;151(1):53-8.

5. Ni N, Wagner RS, Langer P, Guo S. New Developments in the Management of Periocular Capillary Hemangioma in Children. J Pediatr Ophthalmol Strabismus. 2011;48(5):269-76.

6. Frank RC, Cowan BJ, Harrop AR, Astle WF, McPhalen DF. Visual development in infants: visual complications of periocular hemangiomas. J Plast Reconstr Aesthet Surg. 2010;63(1):1-8.

7. North PE, Waner M, Mizeracki A, Mihm JMC. GLUT1: a newly discovered immunohistochemical marker for juvenile hemangiomas. Hum Pathol. 2000;31(1):11-22.

8. Barnes CM, Huang S, Kaipainen A, Sanoudou D, Chen EJ, Eichler GS, et al. Evidence by molecular profiling for a placental origin of infantile hemangioma. Proc Nat Acad Sci USA. 2005;102(52):19097-102.

9. Zimmermann AP, Wiegand S, Werner JA, Eivazi B. Propranolol therapy for infantile haemangiomas: Review of the literature. Int J Pediatr Otorhinolaryngol. 2010;74(4):338-42.

10. Léauté-Labrèze $C$, Dumas de la Roque $E$, Hubiche $T$, Boralevi $F$, Thambo JB, Taïeb A. Propranolol for severe haemangiomas of infancy. N Engl J Med. 2008;358(24):2649-51.

11. Gawley SD, Bingham EA, McGinnity G. Visual outcomes of treated periocular capillary haemangiomas in childhood: a10-year review. Acta Ophthalmol. 2011;89(4):396-401.

12. Boon LM, MacDonald DM, Mulliken JB. Complications of systemic corticosteroid therapy for problematic hemangioma. Plast Reconstr Surg. 1999;104(6):1616-23.

13. Arneja JS, Mulliken JB. Resection of amblyogenic periocular hemangiomas: indications and outcomes. Plast Reconstr Surg. 2010;125(1):274-81.

14. Greinwald JH Jr, Burke DK, Bonthius DJ, Bauman NM, Smith RJ. An update on the treatment of hemangiomas in children with interferon alfa-2a. Arch Otolaryngol Head Neck Surg. 1999;125(1):21-7.

15. Chen TS, Eichenfield LF, Friedlander SF. Infantile hemangiomas: an update on pathogenesis and therapy. Pediatrics. 2013;131(1):99-108.

16. Ni N, Langer P, Wagner R, Guo S. Topical Timolol for Periocular Hemangioma: Report of Further Study. Arch Ophthalmol. 2011;129(3):373-9.

17. Haider KM, Plager DA, Neely DE, Eikenberry J, Haggstrom A. Outpatient treatment of periocular infantile hemangiomas with oral propranolol. JAAPOS. 2010;14(3):251-6.

18. Guo S, Ni N. Topical Treatment for Capillary Hemangioma of the Eyelid Using $\beta$-Blocker Solution. Arch Ophthalmol. 2010;128(2):255-6.

19. Semkova K, Kazandjieva J. Topical timolol maleate for treatment of infantile haemangiomas: preliminary results of a prospective study. Clin Exp Dermatol. 2013;38(2):143-6.

20. Jha AK, Mallik SK, Raihan M. Topical ophthalmic solution in infantile hemangioma. J Postgrad Med. 2012;58(2):163-5.

21. Moehrle $M$, Léauté-Labrèze $C$, Schmidt V, Röcken $M$, Poets $C F$, Goelz R Topical Timolol for Small Hemangiomas of Infancy. Pediatr Dermatol. 2013;30(2):245-9.

22. Calvo M, Garcia-Millán C, Villegas C, Fueyo-Casado A, Burón I. Topical timolol for infantile hemangioma of the eyelid. Int $\mathrm{J}$ Dermatol. 2013;52(5):603-4.

23. Chambers CB, Katowitz WR, Katowitz JA, Binenbaum G. A Controlled Study of Topical $0.25 \%$ Timolol Maleate Gel for the Treatment of Cutaneous Infantile Capillary Hemangiomas. Ophthal Plast Reconstr Surg. 2012;28(2):103-6.

24. Oranje AP, Janmohamed SR, Madern GC, de Laat PC. Treatment of Small Superficial Haemangioma with Timolol 0.5\% Ophthalmic Solution: A Series of 20 Cases. Dermatology. 2011;223(4):330-4. 